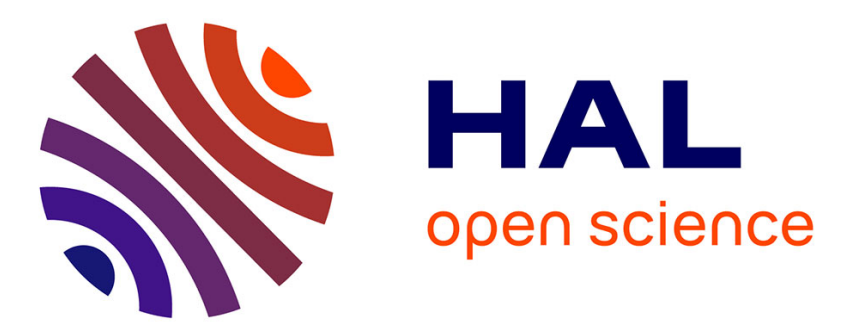

\title{
Instability of the one-dimensional thickness profile at the edge of a horizontal foam film and its Plateau border
}

C. Tregouet, Isabelle Cantat

\section{To cite this version:}

C. Tregouet, Isabelle Cantat. Instability of the one-dimensional thickness profile at the edge of a horizontal foam film and its Plateau border. Physical Review Fluids, 2021, 6 (11), pp.114005. 10.1103/PhysRevFluids.6.114005 . hal-03464485

\section{HAL Id: hal-03464485 \\ https://hal.science/hal-03464485}

Submitted on 14 Dec 2021

HAL is a multi-disciplinary open access archive for the deposit and dissemination of scientific research documents, whether they are published or not. The documents may come from teaching and research institutions in France or abroad, or from public or private research centers.
L'archive ouverte pluridisciplinaire HAL, est destinée au dépôt et à la diffusion de documents scientifiques de niveau recherche, publiés ou non, émanant des établissements d'enseignement et de recherche français ou étrangers, des laboratoires publics ou privés.

\section{(c) (1) $\$$}

Distributed under a Creative Commons Attribution - NonCommerciall 4.0 International 


\title{
Instability of the 1D thickness profile at the edge of a horizontal foam film and its Plateau border
}

\author{
Corentin Trégouët and Isabelle Cantat \\ Univ Rennes, CNRS, IPR (Institut de Physique de Rennes) - UMR 6251, F- 35000 Rennes.
}

(Dated: November 29, 2021)

The thinning of the liquid films separating bubbles in a foam or in a bubbly liquid controls the coalescence process and the foam stability, and is highly relevant in many industrial processes. The spatio-temporal evolution of the film thickness is governed by highly nonlinear equations, the solution properties of which are still mostly unknown. For a flat film in contact with a meniscus at a lower pressure, a classical theoretical solution is the appearance, deepening and widening of a pinched region, uniform along the meniscus. However, film thinning has been shown to be mainly controlled by non-uniform marginal regeneration, for which no clear explanation has been provided to date. In this article we establish experimentally that the theoretical uniform film thickness profile is unstable, even in a horizontal film, and we measure the instability wavelength along the meniscus. We show that the Poiseuille flow can be neglected during the destabilization process, and, taking advantage of this scale separation, we build an original equation set leading to the prediction of the instability wavelength. 


\section{INTRODUCTION}

Liquid foams are complex fluids used in various industrial domains, such as food science, oil recovery, the cosmetic and detergent industry, ore extraction, or fire fighting. In most situations, the foam stability, i.e. its life time, is a key parameter; it is difficult to control or predict because the dynamics of film thinning is still poorly understood. The foam films consist of surfactant solution confined between two gas bubbles and limited laterally by a meniscus, as shown in Figure 1a. Their dynamics belongs to the large class of lubrication flows that are governed by a strong coupling between the shape of the limiting interfaces and the hydrodynamical forces. In the foam-film case, the coupling arises from the Laplace law [1], relating the interface curvature and the pressure: in the meniscus, the curvature is fixed by the bubble diameter and the total volume of foaming solution, yielding a pressure lower than in the air; in the film, the thickness evolves with the flow of solution, generating a non-uniform curvature that gives a complex feedback on the flow. It results into a peculiar dynamics and, depending on the boundary condition at the interfaces $[2,3]$ and on the bubble motion, the meniscus under-pressure may lead to different drainage processes: in particular, the thinnest part of the film may be either the film center [4-6] (Figure 1b), or a pinched region at the film edge [7-10](Figure 1c). In this last case, denoted marginal pinching in the literature, the film thickness profile has been predicted by Aradian, Raphaël and de Gennes [2], assuming the interfaces do not move. This important simplification actually results from two different assumptions: (i) an incompressible interface, which is a limit usually reached in foam films, due to their high interface elastic modulus [11], and (ii) the uniformity of the profile along the meniscus direction, which only results from the symmetry of the problem. These two conditions impose a uniform velocity at the interface, and thus a velocity equal to zero on the whole interface when the film is at rest far from the meniscus. The aim of the paper is to question the assumption (ii) and to investigate the stability of the 1D profile with respect to $2 \mathrm{D}$ perturbations.

(a)

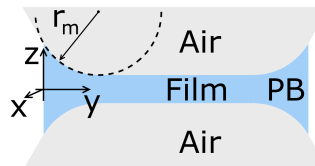

(c)

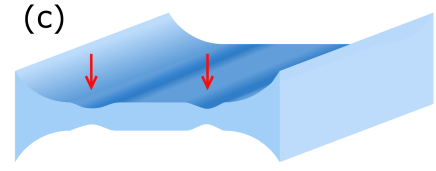

(b)

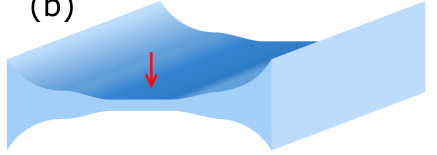

(d)

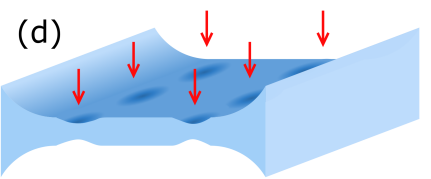

FIG. 1. (a) Sketch of the studied configuration: a flat film of surfactant solution separates two volumes of air (bubbles), and is delimited by two Plateau borders (PB) of uniform radius of curvature $r_{m}$. Under the assumption of a thickness uniform along the $\mathrm{x}$ direction, different cases are known for the drainage depending on the boundary condition at the interfaces: the thinnest part of the film can be located in the center (b) or at the edges of the film (c) In the experiments and in the present modeling, the thickness profile is close to case (c) at short time. However, it does not remains uniform along the $x$ direction at long time, and destabilizes into a periodic pattern $(\mathrm{d})$.

We first recall here the mechanism detailed in [2] leading to the pinched regions close to the meniscus (see Figure 1c). A meniscus (also called a Plateau border) of uniform radius of curvature $r_{m}$ is oriented along $x$ and in contact with a flat immobile film of thickness $2 h_{\infty}$ at large $y$. The film is horizontal, so there are no gravity effects, and it is thick enough for the disjoining pressure to be negligible. The pressure in the film at the position $y$ is thus entirely controlled by the Laplace pressure, which is the product of the interfacial tension $\gamma$ and of the interface curvature. The Reynolds number is negligible and the pressure gradient balances the viscous forces associated with the Poiseuille flow in the film. Due to volume conservation, the flow, which is driven by the thickness profile, also modifies the thickness. This complex feedback loop generates the following dynamics: at the edge between the flat film at atmospheric pressure and the meniscus at a lower pressure, there is a flow from the film to the meniscus. This flow decreases the thickness at the film edge and creates a trough and a new pressure distribution along the film, with a low pressure at the bottom of the trough and a high pressure at its border. The subsequent fluxes result in a widening and a deepening of the trough. The modeling discussed in section IV yields at long time to a scale invariant film profile, with scaling laws for the characteristic width $w$ of the pinched region and for the minimal thickness $h_{\min }$ of the profile as a function of time $t$ :

$$
w \sim r_{m} \cdot\left(\frac{t}{\tau}\right)^{1 / 4}
$$


and

$$
h_{\text {min }} \sim \frac{h_{\infty}^{2}}{r_{m}} \cdot\left(\frac{t}{\tau}\right)^{-1 / 2}
$$

with $\tau$ being a visco-capillary time such as $\tau=3 \eta r_{m}^{4} /\left(\gamma h_{\infty}^{3}\right)$ where $\eta$ is the viscosity of the liquid. In this mechanism, the flow is $1 \mathrm{D}$, and the thickness profile remains uniform in the direction parallel to the meniscus.

This process is at the origin of the dimple shapes often observed in draining films [12]. In some cases, the pinched region is reported to destabilize into a necklace of thin spots [13-15], and this instability then evolves into a nonuniform flow dynamics along the meniscus $[1,16,17]$, which controls the subsequent film drainage and its life time [10, 18-20]: regions of the film with a small thickness are emitted from the menisci, while thicker parts of the film flow into the menisci. In the presence of gravity, the thin regions migrate by buoyancy to the top of the film. The localization of this phenomenon at the margin of the film yielded the term of marginal regeneration [1]. The identification of the parameters controlling the destabilization process, and the prediction of the initial wavelength are open questions in the literature.

Symmetry breaking induced by the disjoining pressure [21] or by an increase of the tension far from the meniscus, due to evaporation in binary mixture [22], has been observed. In this paper, we show that the film profile destabilization occurs even in the absence of gravity or disjoining pressure and that it can be explained by capillary effects only. We produce $600 \mathrm{~nm}$ thick horizontal film at rest, in contact with a meniscus. A thinner region appears along the meniscus, initially uniform by translation along the meniscus, and this pinched region destabilizes into quasi circular spots of thin film separated by a well defined wavelength. Revisiting the classical lubrication equations, we show that this destabilization occurs by a simple reorganization of the film elements in the plane of the film, driven by the interface area minimization, under the novel constraint that the film thickness distribution must be preserved. Under well-controlled approximations, we show that the instability is entirely decoupled from the underlying drainage process, which allows us to predict the scaling laws for the instability wavelength, in qualitative agreement with our observations. The wavelength later increases, due to a coarsening process studied in [17].

\section{EXPERIMENTAL SET-UP}

\section{A. Physico-chemical properties of the foaming solution}

The foaming solution consists of water, with $15 \% \mathrm{w}$ glycerol, $5.6 \mathrm{~g} / \mathrm{L}$ of Sodium Dodecyl Sulfate (cmc: $2.36 \mathrm{~g} / \mathrm{L}$ ), and $0.8 \mathrm{~g} / \mathrm{L}$ fluorescein. The resulting viscosity is $1.5 \mathrm{mPa} . \mathrm{s}$ and the surface tension is $\gamma=40 \mathrm{mN} / \mathrm{m}$. The surface shear viscosity is not known with a high degree of precision, and the values reported in the literature show a large dispersion [23]. Here we assume $\eta_{s}=6 \cdot 10^{-8} \mathrm{~kg} \cdot \mathrm{s}$, following [24]. The hygrometry is not controlled, and the evaporation may lead to a slow increase of the initial concentrations. A thickness decrease rate of $30 \mathrm{~nm} / \mathrm{s}$ may be deduced from [25], for horizontal interfaces of surfactant solution of comparable area, and for a relative humidity of $50 \%$. This should not play any role during the destabilization time.

\section{B. Mechanical and optical devices}

Our experimental set-up is similar to the set-up used in [17], in which the experimental details are given. A deformable frame holds three rectangular, flat, foam films connected by a free meniscus (or Plateau border). The film of interest is horizontal, of constant dimension $L_{x}$ in the $x$ direction and variable dimension $L_{y}$ in the $y$ direction (see Fig. 2). Its dynamics is monitored through two cameras. A Photron $S_{4}$ fast camera, perpendicular to the film, records the fluorescent signal thanks to a blue LED illumination and a dichroic filter (1000 fps, $5 \mu \mathrm{m} /$ pixel). The film thickness, as well as the meniscus width, are measured from these images. A Resonon Pika L spectral camera provides a better determination of the height profile along a line perpendicular to the free meniscus, by specular reflection of a halogen light forming an angle of $48^{\circ}$ with the vertical direction (100 fps, $2.5 \mu \mathrm{m} /$ pixel).

\section{Film preparation}

The three motor displacements are tuned to produce a horizontal film close to the ideal initial conditions discussed in ref. [2]: a film of uniform thickness and vanishing interface velocity, in contact with a static meniscus. Toward this end, the frame is plunged into the solution, producing the three initial films. Then the horizontal film is stretched and 


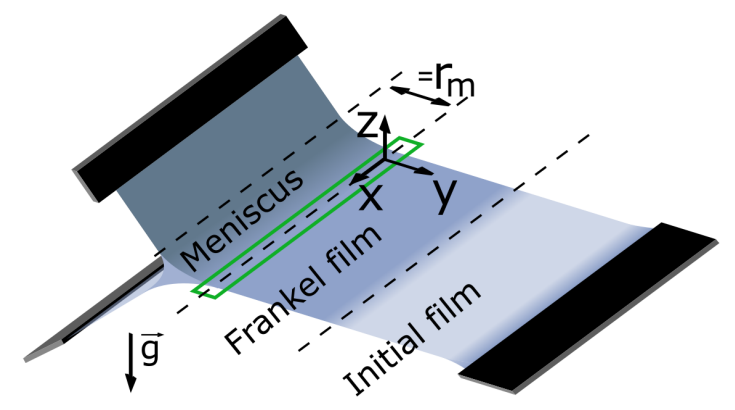

FIG. 2. Sketch of the experimental setup. Three films extend between a free meniscus and mobile frames, and the film of interest is the horizontal one in the xy-plane. To produce a reproducible initial condition, a piece of film of controlled thickness (of the order of one micron), denoted as the Frankel film, is extracted from the free meniscus by stretching the horizontal film. The green rectangle represents the area of interest pictured in Figure 4. The meniscus on the mobile frame (on the right) behaves similarly but is not studied.

(a)

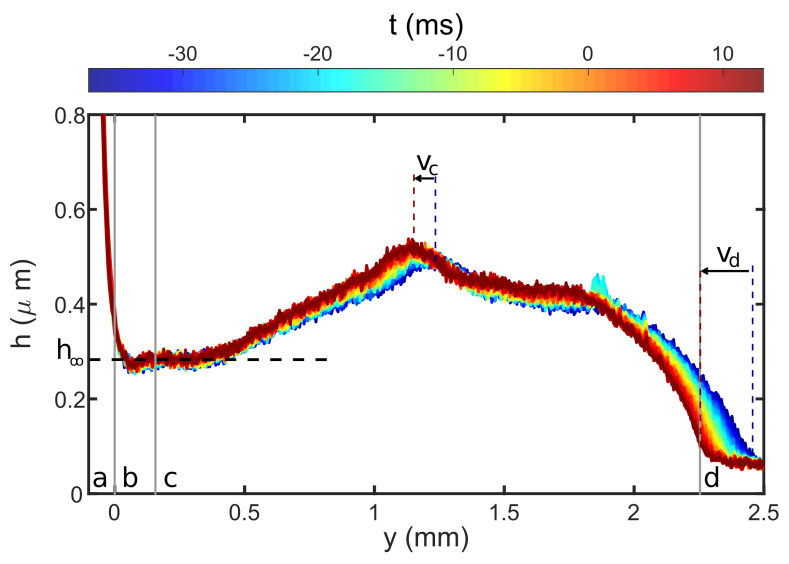

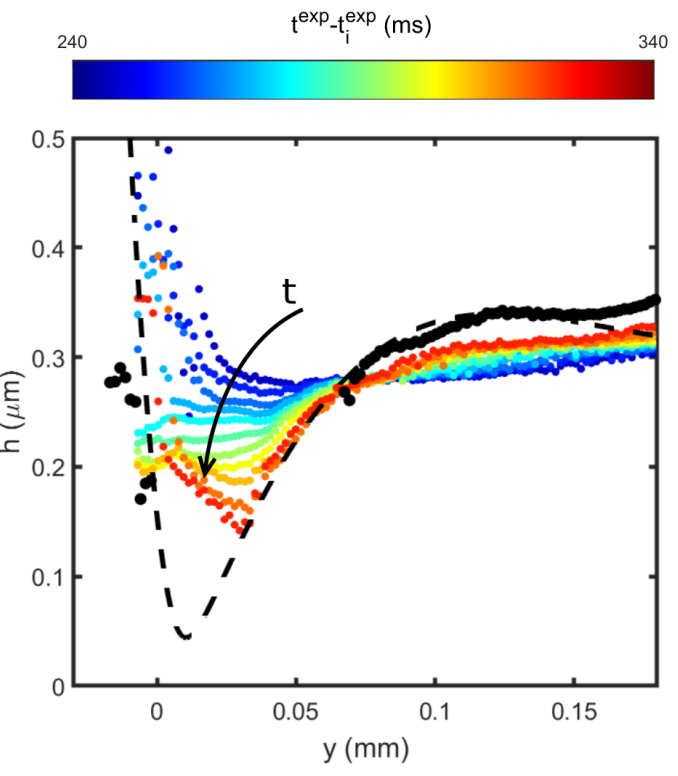

(b)

FIG. 3. (a) Film profile ( $h$ is the half thickness) from fluorescence measurement calibrated through the spectral camera, close to the destabilization time at $t=0$ (in this example $t_{i}=-450 \mathrm{~ms}$ ). Areas a, b, c and d respectively correspond to the meniscus, the pinched region, the Frankel film, and the initial thin film. Two characteristic material points can be followed during this time range and move at the velocities $v_{c}=1.6 \mathrm{~mm} / \mathrm{s}$, and $v_{d}=4.0 \mathrm{~mm} / \mathrm{s}$ indicated in the figure, showing the film compression. (b) Zoom on the 1D film profiles obtained with the spectral camera for the same experiment, before the destabilization. The colored profiles are recorded from $t=-210 \mathrm{~ms}$ (dark blue) to $-110 \mathrm{~ms}$ (red) every $10 \mathrm{~ms}$ and the black one show the measurable part of the profile at $t=0 \mathrm{~ms}$.

the lateral films are compressed by moving the motors at a speed of $50 \mathrm{~mm} / \mathrm{s}$. The tension increases in the horizontal film and some new film is extracted from the meniscus, following the Frankel's law [1]. The thickness of the new film, located between the initial film and the meniscus and denoted as the Frankel's film in the following, is of the order of $600 \mathrm{~nm}$, so that the disjoining pressure is negligible. If the motor is suddenly stopped, the film extraction continues as long as the horizontal film tension is above its steady value. The extraction velocity, and thus the Frankel's film thickness, decreases during the tension relaxation process, and the flat film initial condition is therefore not achieved. A much better result is obtained by moving the motors back by a small amount after the stretching step. This relaxes the film tension and stops the film extraction more rapidly. The boundary between the initial film and Frankel's film (c-d limit in Figure 3) starts receding at the time $t=t_{i}$ defined as the initial time of the experiment. As discussed below, the interface velocity vanishes close to the meniscus at an intermediate time, after the motor stop and before $t_{i}$. The instability of interest occurs typically $0.5 \mathrm{~s}$ later than $t_{i}$. 


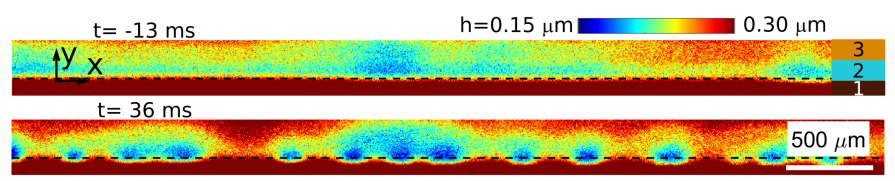

FIG. 4. Film thickness field obtained from the fluorescence intensity before (top, $t=-13 \mathrm{~ms}$ ) and after (bottom, $t=36 \mathrm{ms)}$ the instability onset ( $h$ is the half-thickness). The bars on the right of the top image indicate the meniscus (region 1 , thickness not resolved), the pinched region, of width $w_{p}$ (region 2), and the Frankel's film, of thickness $2 h_{\infty}$ (region 3 ). The region pictured correspond to the rectangle shown in Fig. 2, and the dashed line is the position $y_{p}$ where the profiles of Fig. 5 are measured.

Following two characteristic material points in the film (the point of maximal thickness and the boundary between the Frankel's film and the initial film) we estimate the compression rate during this relaxation as $\dot{\varepsilon}=2 \pm 1 \mathrm{~s}^{-1}$, which is consistent with the compression rate deduced from the thickness map in section IV B. Assuming an homogeneous compression in the film, the velocity, extrapolated at the boundary with the meniscus, is lower (in absolute value) than $0.5 \mathrm{~mm} / \mathrm{s}$. The upper bound for the interface displacement in the pinched region during the $20 \mathrm{~ms}$ of the destabilization process is therefore $10 \mu \mathrm{m}$, i.e. a small fraction of the width of the profile. The best evidence of the vanishing velocity at the meniscus results from the analysis in section IV B. In the following, we thus assume that the interface velocity is vanishing at the meniscus during the destabilization.

The region of interest is located in the Frankel's film, close to the free meniscus. In this domain, the film thickness is uniform at $t_{i}$, of value $2 h_{\infty}$. This thickness as been varied in the range $[0.5,1] \mu m$ and is correlated with the meniscus radius $r_{m}$, which is in the range $[375,570] \mu \mathrm{m}$. From this initial condition, we let the film evolve spontaneously.

\section{Initial film profile characterization}

The film first remains uniform in the $x$ direction and get locally thinner at the boundary between the meniscus and the film. Then, at a critical time, chosen as the reference time $t=0$ (note that this implies $t_{i}<0$ ), the uniformity in the $x$ direction is spontaneously broken and the pinched region begins to destabilize in small circular patches of thin film surrounded by the film of thickness $2 h_{\infty}$ (see Fig. 4). The film half-thickness profiles $h^{\exp }(y, t)$ obtained with the spectral camera at different times between $t=t_{i}$ and the time $t=0$ are shown in Fig. 3 . The profiles at short time are entirely resolved, but when thickness gradients become to large, or the thickness to thin, the interference pattern is lost. In particular, at the destabilization time, the thinnest part of the profile is not measured. Few data points are obtained on the meniscus side, and show a fast increase of the thickness, as expected. This enable us to localize precisely the meniscus. On the film side, the upper part of the profile is resolved.

\section{E. Instability wavelength and destabilization time}

We now focus on the destabilization of the film profile at $t>0$. The fluorescence intensity along a line $y=y_{p}$ close to the meniscus (see Fig. 4) is plotted for different times in Fig. 5. We define $\lambda_{\text {exp }}$ as the wavelength measured on the first profile where an oscillating intensity can be detected. More precisely, in profiles like the one presented in Figure 5, we identify patterns consisting in a few wavelengths that grow simultaneously over time, and we measure the wavelength at the first instant where it can be detected. The resulting measurements are presented as a function of the half-thickness of the flat film $h_{\infty}$ in Figure 6.

\section{THEORY}

We consider a film of half-thickness $h(x, y)$ as schematized in Fig. 7. As in Mysels' et al. model [1], we assume the Gibbs elasticity to be large enough so that the limit of incompressible interface is reached [11, 26]. The interface is therefore seen as a two dimensional incompressible Newtonian fluid, of interface shear viscosity $\eta_{s}$ and of tension $\gamma=\gamma_{0}+\delta \gamma(x, y, t)$, with $\gamma_{0}$ a reference tension, taken as the equilibrium tension of the foaming solution. In this limit, the tension variation $\delta \gamma$ is not related to any thermodynamical property of the interface and it can be seen as the Lagrange multiplier associated with the conservation of the local interface area. This model has been introduced by Mysels et al. [1] to describe the motion of the interfaces defined as 'mobile'. This must not be confused with the 


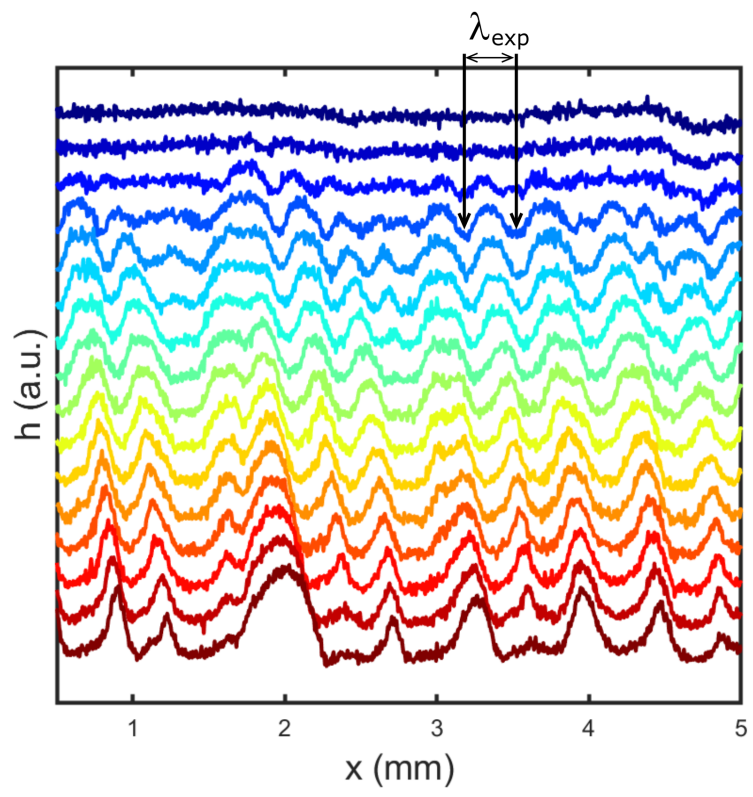

FIG. 5. Intensity profiles along the line $y=y_{p}$ (shown in Fig. 4) recorded every $10 \mathrm{~ms}$, from $t=0$ (top, dark blue) to $t=150$ ms (bottom, dark red). A arbitrary offset is added for the sake of visibility.

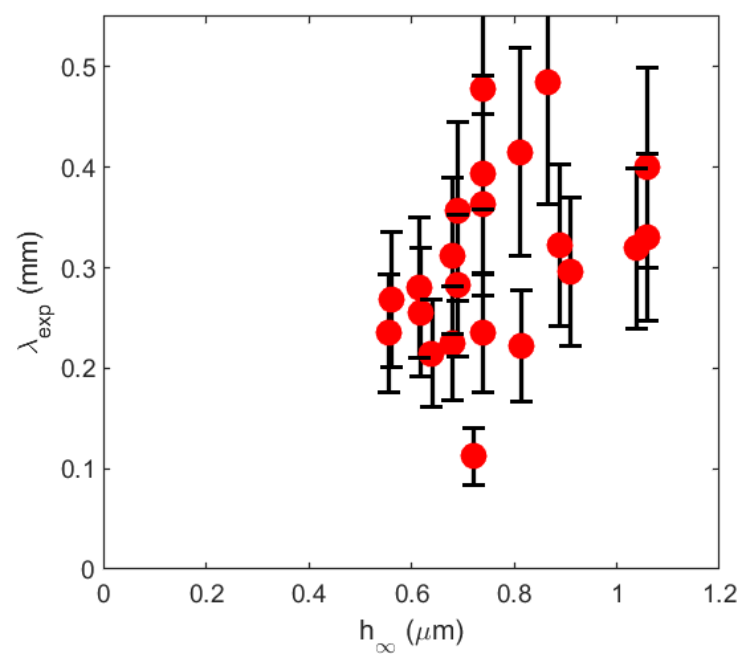

FIG. 6. Measured wavelengths as a function of the thickness of the flat Frankel film.

'rigid' interfaces which follow similar rules, but with an infinite shear viscosity which forbid any recirculation in the film.

\section{A. Lubrication equations}

The lubrication equations governing the bulk velocity $\mathbf{u}(x, y, z, t)$ and the half film thickness $h(x, y, t)$ have been established in $[16,27]$ and are briefly recalled here. They can be derived from the 3D Stokes equation by making a Taylor expansion, using $\varepsilon=h / L$ as a small parameter, with $L$ the characteristic length scale of the thickness variations. At dominant order, the normal velocity is negligible and $\mathbf{u}=\left(u_{x}, u_{y}\right)$; the pressure is uniform across the film and its value is $P(x, y)=-\gamma_{0} \Delta h$, with $\Delta h$ the interface mean curvature (note that $\Delta$ and $\nabla$ are the 2D operators). 


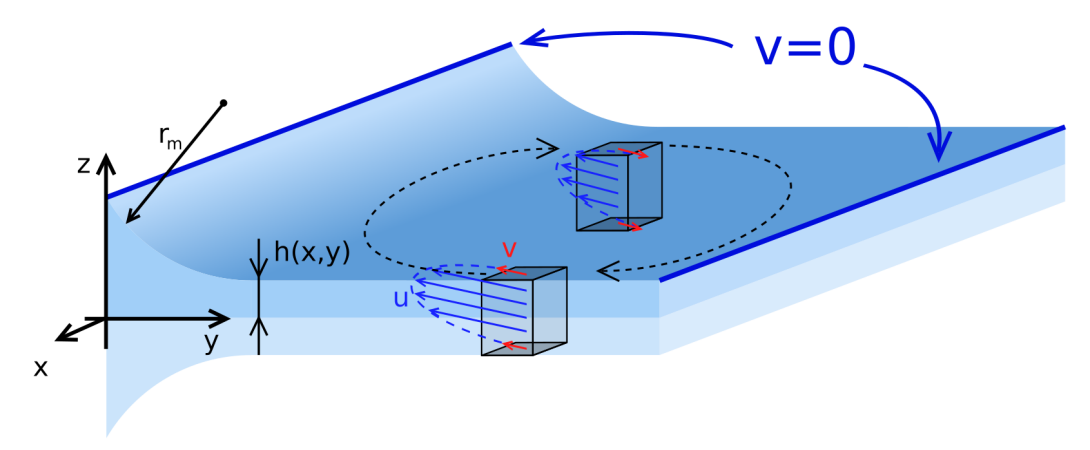

FIG. 7. Notations used in the text and schematic of the most general flow pattern, associated with the equations (3- 5).

The Stokes equation $\eta \partial^{2} \mathbf{u} / \partial z^{2}=\nabla P$ leads to, by successive integrations,

$$
\begin{aligned}
& \frac{\partial \mathbf{u}}{\partial z}=-\frac{\gamma_{0}}{\eta} \boldsymbol{\nabla}(\Delta h) z \\
& \mathbf{u}=-\frac{\gamma_{0}}{\eta} \boldsymbol{\nabla}(\Delta h)\left(\frac{z^{2}}{2}-\frac{h^{2}}{2}\right)+\mathbf{v}, \\
& \mathbf{q}=\int_{0}^{h} \mathbf{u} d z=\frac{\gamma_{0}}{3 \eta} h^{3} \boldsymbol{\nabla}(\Delta h)+\mathbf{v} h
\end{aligned}
$$

The equation (3) is obtained using the symmetry condition in the middle of the film $\partial \mathbf{u} / \partial z(0)=0$ and the equation (4) uses the boundary condition $\mathbf{u}(h)=\mathbf{u}(-h)=\mathbf{v}$.

The mass conservation in the film gives finally

$$
\frac{\partial h}{\partial t}=-\boldsymbol{\nabla} \cdot\left[\frac{\gamma_{0}}{3 \eta} h^{3} \boldsymbol{\nabla}(\Delta h)+\mathbf{v} h\right]
$$
$[16]$ :

The interface velocity $\mathbf{v}(x, y, t)$, is determined from the condition of tangential stress continuity at the interface

$$
\gamma_{0} h \nabla(\Delta h)+\eta_{s} \Delta \mathbf{v}+\nabla \delta \gamma=0
$$

This equation can be seen as the Stokes equation for the interface, modeled as a 2D Newtonian incompressible fluid. The term $\eta_{s} \Delta \mathbf{v}$ represents the viscous forces in the interface, in the limit of small interface curvature and the term $\boldsymbol{\nabla} \delta \gamma$ is the $2 \mathrm{D}$ equivalent of a pressure term, insuring the interface incompressibility. The first term has no 3D equivalent, as it arises from the friction between the interface and the underlying fluid, which is proportional to the bulk velocity derivative, given by eq. 3 .

For an interface element of projected area $d S=d x d y$, the actual area is

$$
d A=\sqrt{1+(\nabla h)^{2}} d x d y \sim d x d y+\frac{1}{2}(\nabla h)^{2} d x d y .
$$

At dominant order, the conservation of $d A$ implies that $d x d y$ must be conserved, leading to the condition $\boldsymbol{\nabla} \cdot \mathbf{v}=0$. This condition can be taken into account by using the stream function $\psi(x, y)$ defined such as $\mathbf{v}=\left(\frac{\partial \psi}{\partial y},-\frac{\partial \psi}{\partial x}\right)$. After derivation of both components of eq. (7), they are combined into

$$
\eta_{s}\left(\frac{\partial^{4} \psi}{\partial x^{4}}+2 \frac{\partial^{4} \psi}{\partial x^{2} y^{2}}+\frac{\partial^{4} \psi}{\partial y^{4}}\right)=\gamma_{0}\left(\frac{\partial h}{\partial x} \frac{\partial \Delta h}{\partial y}-\frac{\partial h}{\partial y} \frac{\partial \Delta h}{\partial x}\right) .
$$

The film dynamics is thus governed by the coupled equations 6 and 9, once initial and boundary conditions have been set. 


\section{B. Solutions with vanishing interface velocities}

Let us first remark that eq. 9 imposes that solutions with vanishing interface velocity verify

$$
\frac{\partial h}{\partial x} \frac{\partial \Delta h}{\partial y}-\frac{\partial h}{\partial y} \frac{\partial \Delta h}{\partial x}=0 .
$$

It follows immediately that any profile uniform along $x$ belongs to this class of solutions, and is thus compatible with the condition $\mathbf{v}=0$.

In this $1 \mathrm{D}$ case, the equations set $(6,9)$ simplifies into the equation $(6)$ only, in its $1 \mathrm{D}$ version and with $\mathbf{v}=\mathbf{0}$. As discussed in the introduction, this case has been solved by Aradian et al. [2] to predict the evolution of a foam film in contact with a straight meniscus, assuming a priori a solution uniform along the meniscus. This situation corresponds to our experimental case, before the instability onset, and the corresponding equation is solved numerically in section IV A to analyze our initial condition.

The condition $\mathbf{v}=\mathbf{0}$ is also compatible with axisymmetric shapes. Indeed, eq. (10) can be written in polar coordinates $(r, \theta)$ as

$$
\left(\sin \theta \frac{\partial h}{\partial r}+\frac{1}{r \sin \theta} \frac{\partial h}{\partial \theta}\right)\left(\cos \theta \frac{\partial \Delta h}{\partial r}-\frac{1}{r \sin \theta} \frac{\partial \Delta h}{\partial \theta}\right)=\left(\cos \theta \frac{\partial h}{\partial r}-\frac{1}{r \sin \theta} \frac{\partial h}{\partial \theta}\right)\left(\sin \theta \frac{\partial \Delta h}{\partial r}+\frac{1}{r \sin \theta} \frac{\partial \Delta h}{\partial \theta}\right)
$$

which is verified for any function $h(r)$ invariant with $\theta$.

If such symmetries are a priori assumed in a foam film, the condition $\mathbf{v}=0$ at the interface is automatically verified: the condition of 'rigid interface', valid at large interface shear viscosity, is artificially recovered, without any assumption on the interface shear viscosity value. In contrast, for a generic profile $h(x, y)$, the hydrodynamic problem does not have any solution at vanishing interface velocity and the equation (9), involving $\eta_{s}$, must be solved.

\section{The sliding puzzle dynamics}

At this stage, we propose the key approximation of the paper, based on a scale separation between the Poiseuille flux, damped by the bulk viscosity and the flux due to the in-plane recirculation, damped by the interface viscosity.

Assuming that the thickness and velocity variations occur on the characteristic length scale $L$ in both directions $x$ and $y$, the comparison of the two terms of eq. (9) imposes the scaling of the interfacial velocity $v \sim\left(\gamma_{0} h / \eta_{s}\right) h / L$. We can then substitute this scaling in eq. (6) and compare the fluxes associated with the Poiseuille flow and to the recirculation at velocity $v$. The first one is much smaller than the second one if $L \gg L^{s p d}$, with

$$
L^{s p d}=h \sqrt{\frac{\eta_{s}}{h \eta}}
$$

a critical length, of the order of $10 \mu \mathrm{m}$ if $h \sim 1 \mu \mathrm{m}$ and $\eta_{s}=6 \cdot 10^{-8} \mathrm{~kg} \cdot \mathrm{s}^{-1}$. The small value of $L^{s p d}$ makes such dynamics, hereafter denoted as the sliding-puzzle dynamics, relevant for a large class of dynamical problems in foam films. It leads to original and simplified equations of motion, which may allow us to solve problems otherwise difficult to address.

In such regime, eq. (6) becomes

$$
\frac{\partial h}{\partial t}=-\nabla \cdot(\mathbf{v} h)=-\mathbf{v} \cdot \nabla h .
$$

The bulk velocity is homogeneous across the film, equal to $\mathbf{v}$, so an elementary volume $d \Omega=2 h d S$, denoted a film element in the following, is a closed material system of constant volume. The projected area $d S$ is conserved (at leading order in $h / L$ ) and thus $h$ is conserved too and behaves as a passive tracer. The position of a given element in the film, and its shape, may nevertheless vary, one element replacing the other in the film plane, as in a sliding puzzle.

We define the film half-thickness distribution as

$$
\hat{S}\left(h_{0}\right)=\int_{S} H\left(h_{0}-h(x, y)\right) d x d y,
$$

with $H$ the Heavyside function, and $\hat{S}\left(h_{0}\right)$ the projected area of the domain having a half-thickness smaller than $h_{0}$, in the domain $S$ of interest. During a sliding-puzzle dynamics, this distribution is conserved, as $h$ is conserved for each element. However the motion modifies the thickness gradients and induces interface area variations of the order of $(\nabla h)^{2}$. In the following we show that these higher order area variations generate the driving forces for spontaneous in-plane reorganizations of the film. 


\section{Energy balance}

When the Poiseuille flow is negligible, the dissipation in the bulk can be neglected in the energy balance of a domain $\Omega$ of the film. Moreover, if we assume a vanishing interface velocity at the boundary of the system, the tension forces related to $\delta \gamma$ do not provide power to the system. The energy balance, to be derived just below, is thus simply

$$
\gamma_{0} \frac{d A}{d t}+D_{s}=0
$$

with $D_{s}$ the viscous dissipation induced in an interface by the flow.

Despite the apparent simplicity of this equation, rigorously writing the energy balance in capillary flows is not straightforward, as discussed in [28], even for clean interface with vanishing elasticity. Eq. (15) can be established from a scalar product between eq. (7) and the velocity field $\mathbf{v}$, integrated spatially. Using the assumption that $\mathbf{v}$ is vanishing at the integration domain boundary and that eq. (13) applies, the three terms in eq. (7) are computed using several times the relation $\int \mathbf{A} \cdot \boldsymbol{\nabla} B=-\int B \operatorname{div} \mathbf{A}$, valid when $\mathbf{A}$ or $B$ is vanishing at the boundary.

$$
\begin{aligned}
I_{1} & =\int_{S} \gamma_{0} h \boldsymbol{\nabla}(\Delta h) \cdot \mathbf{v} d x d y \\
& =-\gamma_{0} \int_{S} \Delta h \mathbf{v} \cdot \boldsymbol{\nabla} h d x d y, \\
& =\gamma_{0} \int \boldsymbol{\nabla} h \cdot \nabla(\mathbf{v} \cdot \nabla h) d x d y, \\
& =-\gamma_{0} \int \boldsymbol{\nabla} h \cdot \nabla\left(\frac{d h}{d t}\right) d x d y, \\
& =-\gamma_{0} \frac{d A}{d t} . \\
I_{2}= & \int_{S} \eta_{s} \Delta \mathbf{v} \cdot \mathbf{v} d x d y, \\
= & -\eta_{s} \int_{S}\left(\left(\nabla v_{x}\right)^{2}+\left(\nabla v_{y}\right)^{2}\right) d x d y, \\
= & -D_{s},
\end{aligned}
$$

and finally

$$
I_{3}=\int_{S} \boldsymbol{\nabla} \delta \gamma \cdot \mathbf{v} d x d y=0
$$

which leads to eq. (15).

In the limit $L \gg L^{s p d}$, and at the order 2 in $h / L$, the film in-plane reorganizations are entirely decoupled from the bulk properties. They are driven by the area minimisation and damped by the interface viscosity; they are however governed by the Marangoni equation (7), which involves the friction on the bulk (first term) and the tension gradient (third term).

\section{E. The symmetric increasing rearrangement and the Pólya-Szegö inequality}

We deduce from eq. (15) that the stable shapes are minimal surfaces under the constraint of conserved area distribution. The Pólya-Szegö inequality [29, Chap. 3] discussed below implies that the most stable profile in this situation is the axisymmetric one.

This inequality applies to an initial film profile $h(x, y)=h_{\infty}-\delta h(x, y)$ (with $\delta h>0$ defined in the whole plane) under the assumption that $(\delta h)^{2}$ is integrable, as well as the square of its gradient. Note that this excludes the $x$-invariant film profiles.

Let us define the Schwarz symmetric increasing rearrangement $h^{s}(r)$ of the initial film profile $h(x, y)$ as the unique axisymmetric shape that has the same half-thickness distribution $\hat{S}$ and that increases with the distance $r$ to the origin. The profile $h^{s}$ is simply obtained by a rearrangement of the initial film elements of $h$, from the thinnest at 

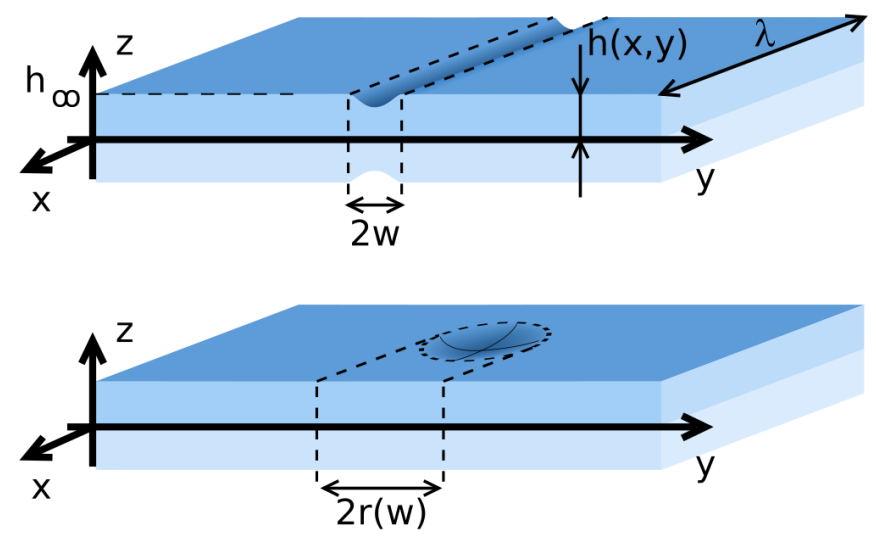

FIG. 8. The Gaussian profile in the domain $\mathcal{P}_{\lambda}$ (top) and its reorganized axisymmetric shape (bottom).

small $r$, to the thickest at larger $r$, in concentric domains. It is an axisymmetric pinched region in the middle of a flat film of asymptotic thickness $h_{\infty}$.

The Pòlya-Szegö inequality states that

$$
\int(\nabla h)^{2} d x d y \geq \int\left(\nabla h^{s}\right)^{2} d x d y .
$$

Using the expression of the film area eq. (8), this inequality proves that among all the shapes accessible by a sliding puzzle dynamics from the initial shape $h$, the symmetric increasing rearrangement $h^{s}$ of $h$ has the smallest area and is thus the most stable shape. This theorem ensures the global stability of a single axisymmetric increasing film profile $h(r)$, for the dynamics of interest. It can be extended to the metastability of a set of such axisymmetric profiles, as long as the pinched regions are separated from each other by a flat film of reference thickness $h_{\infty}$.

The case of an $x$-invariant film profile cannot be directly discussed using the same tool, as it does not belong to the allowed class of profiles. However a direct comparison between the energy of an $x$-invariant pinched region and the energy of a set of isolated axisymmetric pinched regions can be done: if the energy of the later profile is lower, the spontaneous destabilization of the $x$-invariant profile towards this metastable solution is possible, otherwise it is not.

\section{F. Example of Gaussian profiles and scaling law}

In this section, we consider the simple case of a infinite flat foam film, with a thinner part in the vicinity of the $x$ axis. We assume the thickness profile to be uniform in the $x$ direction and Gaussian in the $y$ direction. This differs from our experimental geometry, but provides an illustrative and analytically solvable example. The aim is to compare the interfacial energy of this shape with the energy of shapes, non-uniform in the $x$ direction and in which the thinner part of the first film has been rearranged into a necklace of locally axisymmetric thin patches, with conservation of the thickness distribution. We show that, depending on the wavelength, these non-uniform shapes may be more or less stable than the first one.

Consider an $x$-invariant Gaussian pinched region of width $w$ and depth $h_{0}$

$$
h_{l i n}(x, y)=h_{\infty}-h_{0} e^{-y^{2} /\left(2 w^{2}\right)},
$$

and a band of film $\mathcal{P}_{\lambda}$ of length $\lambda$ in the $x$ direction and spanning from $y=-\infty$ to $+\infty$.

The excess area of the piece of film located in $\mathcal{P}_{\lambda}$, with respect to a flat film in the same domain, is, at dominant order:

$$
\delta A_{l i n}=\lambda \int_{-\infty}^{\infty} \frac{1}{2}\left(\frac{\partial h}{\partial y}\right)^{2} d y=\lambda \frac{h_{0}^{2} \sqrt{\pi}}{4 w} .
$$

An axisymmetric profile $h_{a x i, \lambda}$ is then produced by a spatial reorganization of the film elements located in $\mathcal{P}_{\lambda}$. For a given $y$, all the film elements with a thickness smaller than $h_{\text {lin }}(y)$ are in a rectangular domain of width $2 y$ and length $\lambda$, and hence of projected area $2 \lambda y$. After rearrangement, this rectangle becomes a circular domain of the same area, and hence of radius $r(y)$ such as $\pi r(y)^{2}=2 \lambda y$. 
Before and after rearrangement, the maximum thicknesses are at the edge of the domains, and therefore:

$$
\begin{gathered}
h_{a x i, \lambda}(r(y))=h_{l i n}(y)=h_{l i n}\left(\pi r(y)^{2} /(2 \lambda)\right) \\
h_{a x i, \lambda}(r)=h_{\infty}-h_{0} e^{-\pi^{2} r^{4} /\left(8 \lambda^{2} w^{2}\right)} .
\end{gathered}
$$

The excess area of this shape is

$$
\delta A_{a x i, \lambda}=\pi \int_{0}^{\infty}\left(\frac{\partial h_{a x i, \lambda}}{\partial r}\right)^{2} r d r=\pi h_{0}^{2},
$$

and is thus more favorable than the initial $1 \mathrm{D}$ profile if

$$
\lambda>\lambda_{G}^{*}=4 \sqrt{\pi} w .
$$

The same transformation is made for every band of width $\lambda$, leading to a set of axisymmetric pinched regions. They are not strictly isolated, but the obtained pinched regions can be distributed in the $(x, y)$ plane to ensure that they are separated by a film of thickness as close as we want to $h_{\infty}$.

For example, if the axisymmetric shape obtained for $\lambda_{G}^{*}$ has its center of symmetry in the middle of $\mathcal{P}$, its value at the boundary of $\mathcal{P}$ is

$$
\begin{aligned}
h_{\infty}-h_{a x i}\left(r=\lambda_{G}^{*} / 2\right) & =h_{0} e^{-\pi^{2}\left(\lambda_{G}^{*}\right)^{2} /\left(2^{7} w^{2}\right)} \\
& =h_{0} e^{-\pi^{3} / 8}=0.02 h_{0}
\end{aligned}
$$

As the asymptotic thickness is almost recovered, this axisymmetric shape can be restricted to the domain $\mathcal{P}_{\lambda}$ without significant modification of $\hat{S}$. It can then be used as an elementary pattern to built a periodic film profile of wavelength $\lambda$, with the axisymmetric pinched regions distributed along a straight line. This remark is valid for any $\lambda>\lambda_{G}^{*}$.

Finally, a Gaussian profile, uniform in the $x$ direction and of width $w$, can destabilize into a row of axisymmetric spots if the associated wavelength is larger than $\lambda_{G}^{*}=4 \sqrt{\pi} w$. These rows of axisymmetric Gaussian spots are metastable shapes, which total energy (per unit length in the $x$ direction) decreases with the wavelength. This criteria is of purely geometric nature and the smallest allowed wavelength is proportional to the pinched region width, with a simple numerical prefactor. Note that the dynamics has been entirely disregard in this analysis, so this stability criteria only provides a necessary condition for the destabilization and $\lambda_{G}^{*}$ is a lower bound for the observed wavelength.

This result can be extended to an arbitrary profile, characterized by a depth $h_{p}$ and a width $w_{p}$, using scaling analysis. Indeed, the excess area of a length $\lambda$ of the pinched region is $\delta A_{l i n} \sim \lambda w_{p}\left(h_{p} / w_{p}\right)^{2}=\lambda h_{p}^{2} / w_{p}$ whereas the for the corresponding axisymmetric shape, the gradients are built on the radius of the disc $r_{p}=\sqrt{\lambda w_{p}}$ so $\delta A_{a x i, \lambda} \sim \lambda w_{p}\left(h_{p} / r_{p}\right)^{2}=h_{p}^{2}$. The two excess areas are thus equal if $\lambda \sim w_{p}$, which provide the scaling of the instability wavelength.

\section{COMPARISON WITH EXPERIMENTAL DATA}

The experimental observations can now be rationalized, on the basis of the theoretical arguments developed in section III. When the interfacial velocity vanishes, the film begins to get thinner along the mensicus. This slow dynamics results from a Poiseuille flow, without velocity at the interface, and is governed by eq. (6), in its $1 \mathrm{D}$ version. It leads to the $x$-invariant film profile that eventually destabilizes at $t=0$. This first step is discussed in section IV-A. We then address the destabilization process, which involves non-zero interfacial velocities, 2D recirculation at large scale and a negligible contribution of the Poiseuille flow. This second step obeys the simplified equation (15), based on an approximation whose experimental validity is checked in section IV-B. Finally, the unstable wavelengths and associated growth rates are discussed in IV-C and D.

\section{A. The 1D film profile}

We first discuss the $x$-invariant dynamics occurring between the time $t_{i}<0$ and the time $t=0$ of destabilization. In the $x$-invariant case, the equation (6) becomes

$$
\frac{\partial h}{\partial t}=-\frac{\partial}{\partial y}\left[\frac{\gamma_{0}}{3 \eta} h^{3} \frac{\partial^{3} h}{\partial y^{3}}+v_{y} h\right] .
$$



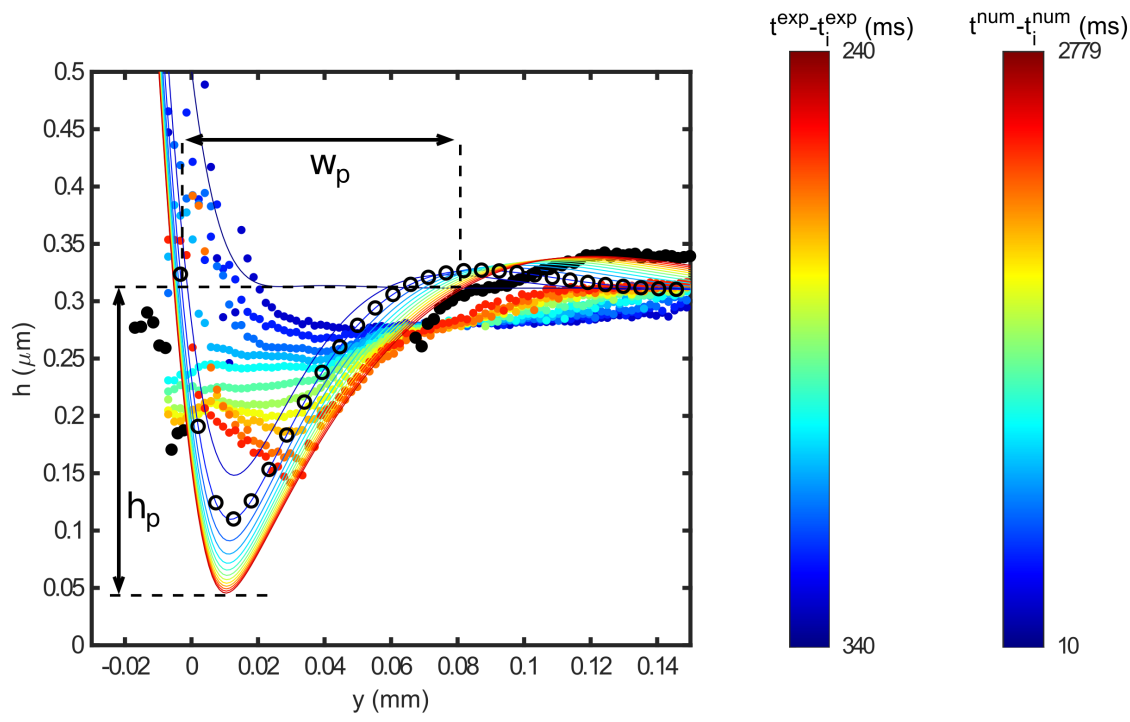

FIG. 9. Film profile evolution with time (in its $x$-invariant regime). The colored symbols are the experimental profiles (same data as in Fig. 3(b)). The lines are the numerical profiles obtained by solving eq. (28) ; the black empty circles underline the numerical profile corresponding to the same film age as the experimental profile shown in black. The two color bars indicate the time with respect to the initial time $t_{i}$ for the numerical and experimental results. The last numerical profile, in red, is $h^{\text {num }}(y)$.

This equation is solved numerically using a Matlab code, with the following assumptions. At small $y$ we impose the measured film curvature $1 / r_{m}$. We first impose a positive velocity $v_{y}$ (oriented toward the film) to prepare a uniform film: using Frankel's theory [1], we choose $v_{y}$ so that the film converges toward a steady film of half-thickness $h_{\infty}$, with $h_{\infty}$ the value measured experimentally at the instability onset. In order to compare our experimental profile with the Aradian's prediction, the interface velocity is set to 0 at the time defined as the time $t_{i}^{\text {num }}$, and we let the shape evolve as a function of the numerical time $t^{\text {num }}$. The successive shapes we obtain are plotted in Fig. 9, with a color code indicating the film age. We explored a larger time range in the simulation than in the experiment, so the two color codes differ.

The dynamics of pinching is qualitatively reproduced, but without quantitative agreement, especially at short time. At long time, the sides of the profile are reproduced by the simulation: a very fast thickness increase at the meniscus side, and a milder slope on the film side. However, the position of the minimum is not captured, and the dynamics is faster in the experiment than in the simulation. We explain this by the fact that, at short time, we are not able to stop the interface instantaneously and some film must be extracted at a slower velocity just before the Frankel's film boundary switch direction at $t_{i}$ (see section II C). Consequently, during few tens of milliseconds before $t_{i}$, instead of pinching a film at rest (Aradian's case [2]), we are extracting a film thinner than $2 h_{\infty}$ from the meniscus (Frankel's case, [1]). The time shift between the experimental and numerical dynamics is thus likely due to the different conditions imposed at short time. Note also that the numerical pinched region width evolves as $t^{1 / 4}$ and because of this small power law, a small width variation is related to a large time variation.

The profile is expected to relax toward the Aradian's solution at long time and indeed the numerical and experimental shapes become closer with time. We thus use the numerical shape to rebuilt the missing part of the experimental profile at the instability onset. In this aim, we let the numerical film evolve until the best agreement is reached with the measurable part of $h^{e x p}(y, t=0)$. In Fig. 9, the resulting profile $h^{\text {num }}(y)$ is the last numerical profile, which fits best the two sides of the unstable profile. It is used to determine the geometrical properties of the experimental profile. The numerical profile has a small bump between the thinnest part of the film and the flat film, and thus reaches the thickness $h_{\infty}$ at a point located between the pinched region and the bump, which provides a good definition of the end of the pinched region: we define $w_{p}$ as the width of the domain for which $h^{\text {num }}(y)<h_{\infty}$. Similarly, the profile depth $h_{p}$ is defined as $h_{\infty}-h_{m i n}^{\text {num }}$, with $h_{m i n}^{\text {num }}$ the minimum value of $h^{\text {num }}(y)$ (see Fig. 9). These two quantities characterize the experimental unstable profile. Note that the fit of the experimental profile relies on the shape and positions of lateral sides of the pinched region. The width $w_{p}$ is thus a robust experimental quantity, whereas $h_{p}$ relies on the modeling of the pinch. However, we have shown in the section III F that the instability wavelength only depends on $w_{p}$, which makes a better determination of $h_{p}$ much less crucial. 

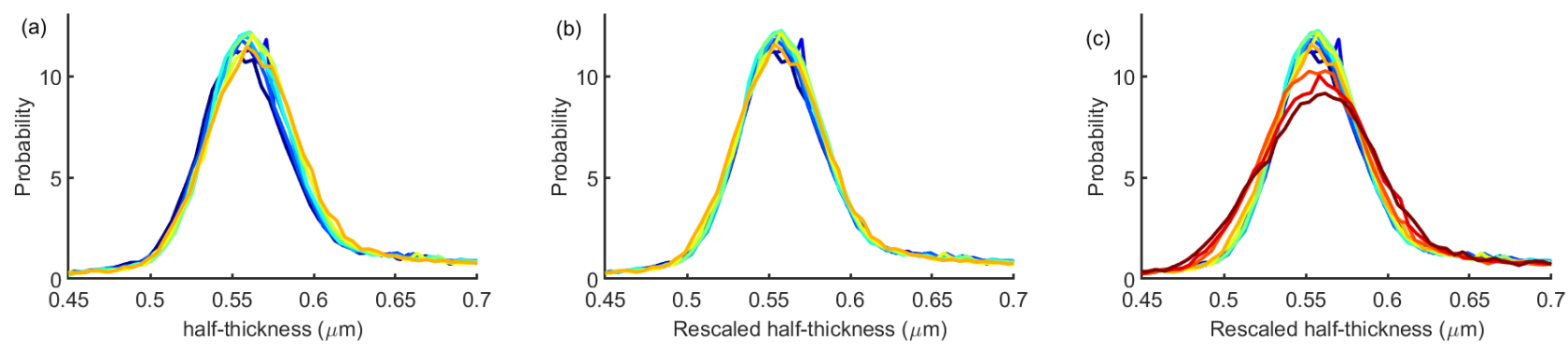

FIG. 10. (a) Probability distribution function of the film half-thickness $h$ in the vicinity of the pinched region, recorded every $5 \mathrm{~ms}$ from $\mathrm{t}=-10 \mathrm{~ms}$ (dark blue) to $\mathrm{t}=25 \mathrm{~ms}$ (orange). (b) Same data, after a global rescaling, taking into account a residual compression of the film. (c) Same data as in $b$, with three additional times 30, 35 and $40 \mathrm{~ms}$.

\section{B. Area distribution}

One key assumption of the model is that the destabilization occurs at constant thickness distribution. To test this assumption, we measured the probability distribution function $P(h)$ of the film half-thickness in the vicinity of the pinched region. For a film of projected area $S_{0}$, this function is defined as $P(h)=\left(1 / S_{0}\right) d \hat{S} /(d h)$, with $\hat{S}$ the area defined by eq. (14). So $P(h) d h$ is the fraction of the image having a half-thickness in the range $[h, h+d h]$. We selected of small ROI restricted to the pinched region in the images of the film, and determined the gray level distribution. This distribution is then normalized and converted to obtain $P(h)$. The distributions obtained before and after the instability onset are shown in Fig. 10(a). The most probable thickness is the asymptotic one $2 h_{\infty}$ and the pinched region contributes to the smaller thicknesses. In Fig. 10(a), we see that the distribution slowly drifts toward larger thicknesses. This indicates a residual compression of the film, which has been corrected in Fig. 10(b) by plotting the distribution of the rescaled half-thickness $h^{r e s}=h /(1+\varepsilon(t))$. We obtain a very good superposition of all the curves in the range $[-10 ; 25] \mathrm{ms}$ if we assume that $\varepsilon$ varies linearly from 0 at $t=-10 \mathrm{~ms}$ (used as arbitrary reference) to 0.015 at $t=25 \mathrm{~ms}$. This indicates that the global film compression during the dynamics is less than $2 \%$ and is thus negligible, as it is assumed in our model.

More importantly the rescaled distributions at small thickness are not modified at the instability onset: in Fig. 10(b), the probability distribution are the same before the onset (negative times) and after (positive times). This validates our assumption that the film profile destabilization is governed by a sliding-puzzle dynamics: it confirms that the Poiseuille flow is negligible and that the interface velocity vanishes at the boundary with the meniscus, as discussed in section II C.

This initial destabilization clearly differs from the coarsening process at later times, that has been shown in [17] to be governed by exchanges of film elements between the film and the meniscus: the first one preserves the thickness distribution function, not the second one. To illustrate this point, we added the thickness distribution functions measured in the time range $[30,40] \mathrm{ms}$ after the onset, in Fig. 10(c). In this regime the film of thickness $2 h_{\infty}$, associated with the maximal area value in Fig. 10 is absorbed by the meniscus, and its probability decreases, while some thin film (of thickness around one micron) is extracted from the meniscus, thus increasing the corresponding probability.

From these graphs, we thus conclude that the film profile destabilization occurs at constant thickness distribution in the time range $[0 ; 25] \mathrm{ms}$, and that the marginal regeneration, involving interface exchanges with the meniscus, begins afterwards.

\section{Unstable wavelength}

The Gaussian example illustrates that the patterns with a wavelength larger than few times the width of the pinched region are more stable than the initial profile, whereas the patterns with shorter wavelength are less stable. This property is of purely geometric nature, and remains true whatever the physical properties of the system, the initial film thickness and the minimal thickness in the pinched region. Assuming that the observed wavelength is close to the shorter accessible wavelength, as is obtained for other classical instabilities damped by viscosity, as the Rayleigh Taylor instability for example, we thus expect the experimental wavelength to verify $\lambda_{\exp }=\alpha w_{p}$, with $\alpha$ a numerical prefactor close to one. 
(a)

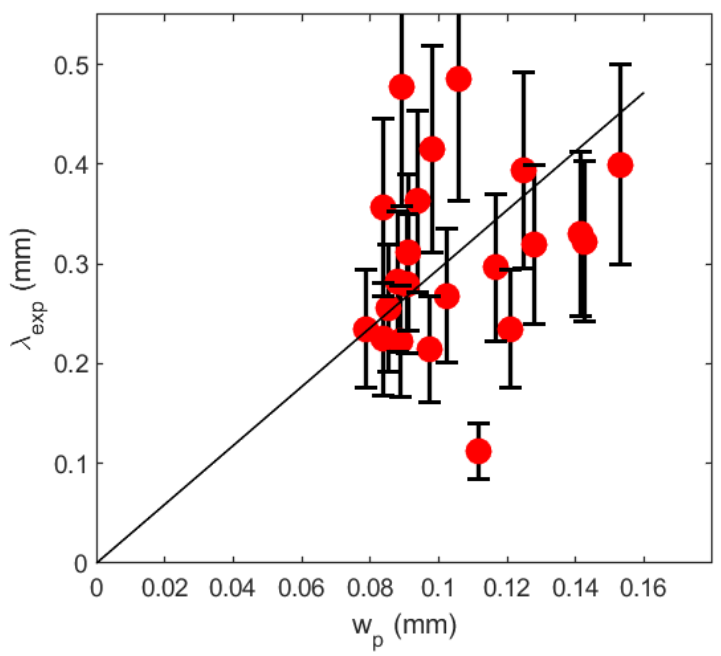

(b)

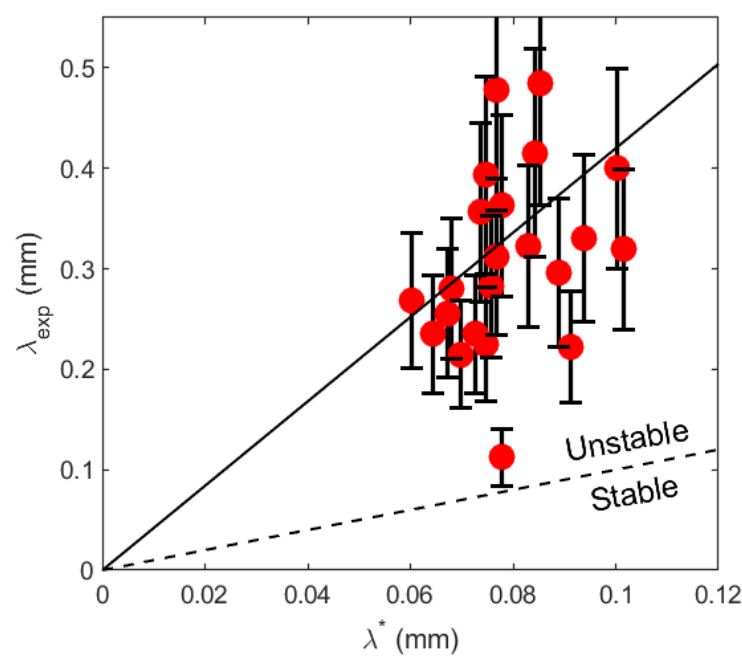

FIG. 11. Experimental wavelength $\lambda_{\exp }$ as a function of the pinched region width (a) and as a function of $\lambda^{*}$ (b). The solid lines are guides for the eyes, of equation $\lambda_{\text {exp }}=2.95 w_{p}$ on the left and $\lambda_{\text {exp }}=4.2 \lambda^{*}$ on the right. The dashed line corresponds to $\lambda_{\text {exp }}=\lambda^{*}$.

This prediction is tested in Fig. 11(a), showing that both quantities are of the same order of magnitude. However, the data dispersion and the narrow range of $w_{p}$ do not permit to test the predicted linear dependency.

The pinched region is not Gaussian, so we determined the critical wavelength $\lambda^{*}$ of the actual film profile from the numerical profile obtained in IV-A. To perform the film reorganization, we consider a piece of pinched region of size $w_{p} \times \lambda$ (the domain $\mathcal{P}_{\lambda}$, between the two dashed lined in Fig. 12(a)).

The film profile in this domain is discretized and a list of pixels is obtained, sorted by their thickness value. These pixels are then reorganized in concentric circles, from the small thicknesses at the center, to the large ones at the perimeter. The rectangle $w_{p}$ thus becomes a disc of same area and of radius $R(\lambda)=\sqrt{w_{p} \lambda} / \pi$ (see Fig. 12(b)). The periodic pattern of the film profile is the whole pattern shown in Fig. 12(b)). The film profile $h^{s}(r)$ is shown in Fig. $12(\mathrm{~d})$.

The excess area of the obtained axisymmetric spot is $\Delta A_{a x i, \lambda}=2 \pi \int_{0}^{R(\lambda)}\left(\partial h^{s} / \partial r\right)^{2} / 2 r d r$. For all our profiles, the condition $\Delta A_{a x i, \lambda}=\Delta A_{\text {lin }}$ is obtained for $\lambda^{*}=(0.7 \pm 0.1) w_{p}$. Note that we verified that $2 R(\lambda)<\lambda$ for $\lambda>\lambda^{*}$, thus allowing us to built the periodic shape without any overlap of the axisymmetric spots for the unstable wavelengths.

The previous analysis evidences that the initial wavelength of the instability must be larger than $\lambda^{*}$. As shown in Fig. 11(b), the observed wavelength verifies $\lambda^{\text {exp }}=(4.2 \pm 1) \lambda^{*}=(2.8 \pm 0.5) w_{p}$, so the fastest wavelength appears to be few times larger than the first unstable one as expected.

\section{Instability growth rate}

Entirely solving the problem would require us to determine the time at which the 1D film profile destabilizes, and the corresponding value of $w_{p}$. This destabilization time is expected to be governed by very different laws if the initial 1D film profile is unstable or only metastable, which remains an open question. The destabilization time of a metastable profile should be controlled by an external noise and by the energy barrier between the metastable initial profile and the more stable final one. In contrast, the destabilization time of an unstable profile (or a metastable with a small enough energy barrier) is expected to be related to the instability growth rate $\tau^{g}$.

The scaling law for the growth rate can be deduced from eq. (15). The instability wavelength $\lambda$ scales as $w_{p}$, so it is the only characteristic length in the film plane. For a piece of film of projected area $S$, the first term scales as $\left(\gamma_{0} / \tau_{g}\right) S\left(h_{p} / w_{p}\right)^{2}$ and the second one as $\eta S\left(1 / \tau_{g}\right)^{2}$ which leads to the prediction

$$
\tau^{g}=\eta_{s} w_{p}^{2} /\left(\gamma_{0} h_{p}^{2}\right) .
$$

A condition for the instability to be observable is that the pinched region is at least as old as $\tau^{g}$, which could provide the scaling for the destabilization time $\left|t_{i}\right|$, if the transition dynamics is limited by the instability growth rate. Experimentally, $\left|t_{i}\right|$ is in the range $[200-600] \mathrm{ms}$ and $\tau_{g} \approx 40 \mathrm{~ms}$. 
(a)
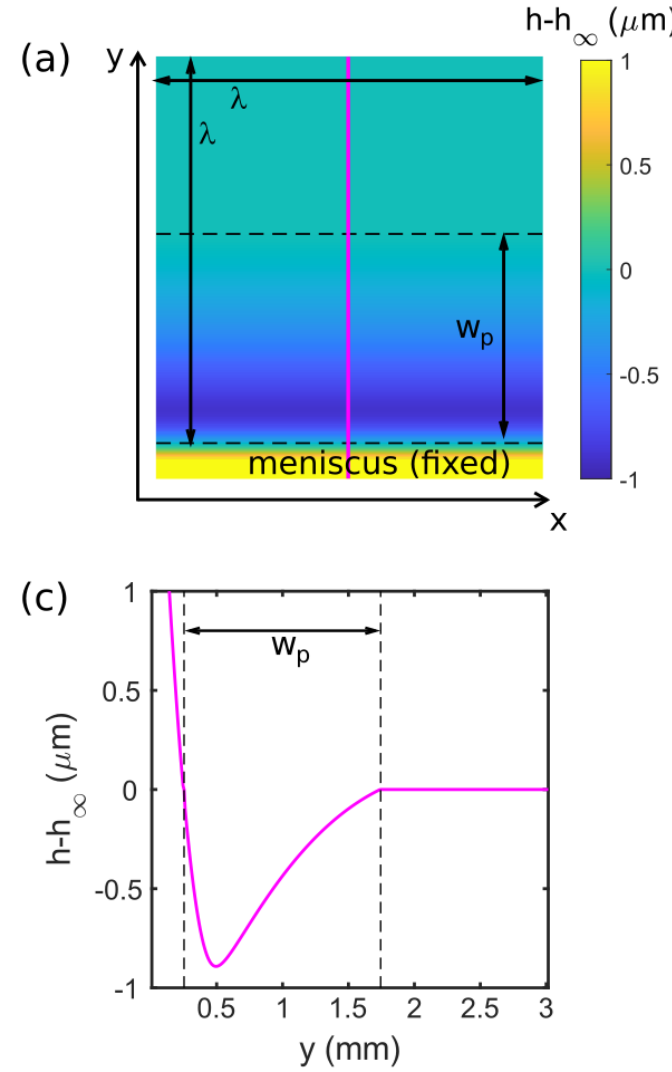
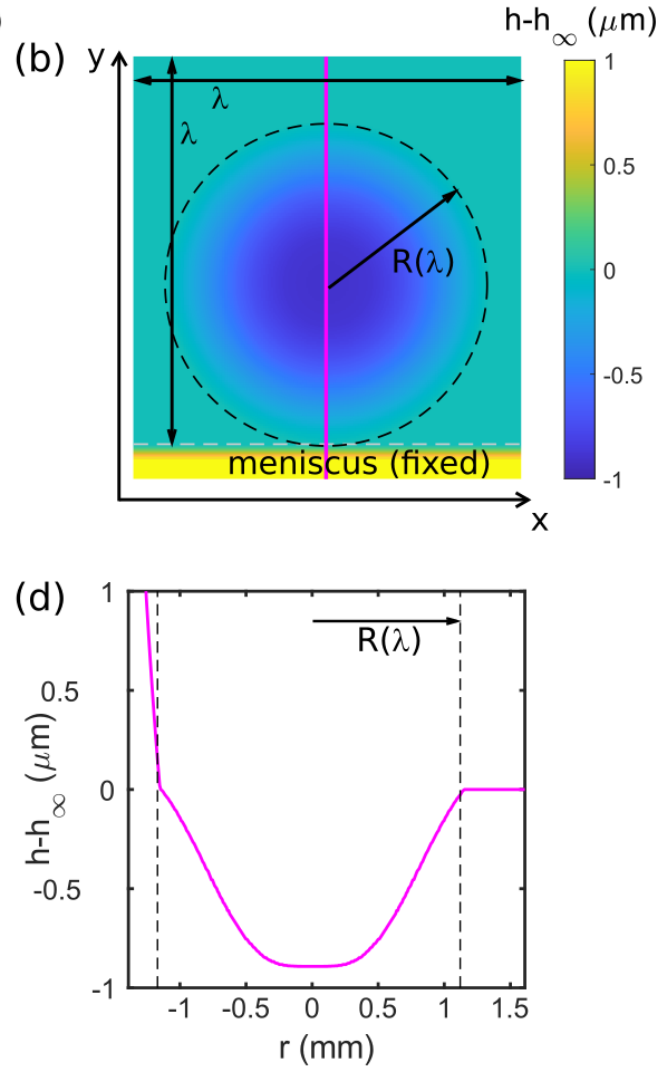

FIG. 12. Example of film profiles before (a) and after (b) the reorganization shown in the $(x, y)$ plane with the color indicating the half-thickness of the film. The reorganized domain $\mathcal{P}_{\lambda}$ is limited by the black dashed lines. The film domain outside $\mathcal{P}_{\lambda}$ is the asymptotic flat film of half-thickness $h_{\infty}$. The thickness profiles along the magenta lines are shown in (c) and (d). The meniscus is shown for clarity (the yellow part in (a) and (b) and the left side of the profile in (c) and (d)) but is not involved in the dynamics.

\section{CONCLUSION}

In this article we evidence experimentally that the 1D film thinning process that arises at the boundary between a meniscus and a one-micron-thick horizontal foam film is unstable. These experimental conditions exclude the gravity and the disjoining pressure as potential driving forces for the instability and indicate its purely capillary origin. We identify a class of foam-film flows governed by the thickness gradients only, and preserving the thickness distribution. We show that in this regime, the stable shape is an axisymmetric rearrangements of the initial shape, and that rows of circular pinched regions are metastable shapes, which energy decreases with the wavelength. The predicted instability wavelength deduced from this theory are compatible with the experimental observations. This article identifies the important role of the film thickness gradients in the foam-film dynamics, at the origin of a purely geometric instability, triggering the marginal regeneration.

\section{ACKNOWLEDGMENTS}

We thank Luc Hillairet and Serge Cantat for enlightening discussions and for reference to the mathematical theory of rearrangement. This project has received funding from the European Research Council (ERC) under the European 
Union's Horizon 2020 research and innovation program (grant agreement No 725094).

[1] K. J. Mysels, K. Shinoda, and S. Frankel, Soap films: Study of their thinning and a bibliography (Pergamon, New-York, 1959).

[2] A. Aradian, E. Raphaël, and P.-G. de Gennes, ""marginal pinching” in soap films," EPL 55, 834 (2001).

[3] P. D. Howell and H. A. Stone, "On the absence of marginal pinching in thin free films," Eur. J. Appl. Math 16, 569-582 (2005).

[4] Raymond R Dagastine, Rogério Manica, Steven L Carnie, DYC Chan, Geoffrey W Stevens, and Franz Grieser, "Dynamic forces between two deformable oil droplets in water," Science 313, 210-213 (2006).

[5] Nicolas Bremond, Abdou R Thiam, and Jérôme Bibette, "Decompressing emulsion droplets favors coalescence," Phys. Rev. Lett. 100, 024501 (2008).

[6] Emmanouil Chatzigiannakis, Peter Veenstra, Dick ten Bosch, and Jan Vermant, "Mimicking coalescence using a pressurecontrolled dynamic thin film balance," Soft Matter 16, 9410-9422 (2020).

[7] Jean Luc Joye, George J Hirasaki, and Clarence A Miller, "Dimple formation and behavior during axisymmetrical foam film drainage," Langmuir 8, 3083-3092 (1992).

[8] B. Dai and L. G. Leal, "The mechanism of surfactant effects on drop coalescence," Phys. Fluids 20, 040802 (2008).

[9] Ivan U. Vakarelski, Rogerio Manica, Xiaosong Tang, Sean J. O'Shea, Geoffrey W. Stevens, Franz Grieser, Raymond R. Dagastine, and Derek Y. C. Chan, "Dynamic interactions between microbubbles in water," PNAS 107, 11177-11182 (2010).

[10] Henri Lhuissier and Emmanuel Villermaux, "Bursting bubble aerosols," J. Fluid Mech. 696, 5-44 (2012).

[11] L. Champougny, B. Scheid, F. Restagno, J. Vermant, and E. Rio, "Surfactant-induced rigidity of interfaces: a unified approach to free and dip-coated films," Soft Matter 11, 2758-2770 (2015).

[12] Derek Y. C. Chan, Evert Klaseboer, and Rogerio Manica, "Film drainage and coalescence between deformable drops and bubbles," Soft Matter 7, 2235-2264 (2011).

[13] Jean-Luc Joye, George J. Hirasaki, and Clarence A. Miller, "Asymmetric drainage in foam films," Langmuir 10, 3174-3179 (1994).

[14] John M. Frostad, Daniele Tammaro, Luciano Santollani, Simone Bochner de Araujo, and Gerald G. Fuller, "Dynamic fluid-film interferometry as a predictor of bulk foam properties," Soft Matter 12, 9266-9279 (2016).

[15] Sébastien Andrieux, Pierre Muller, Manish Kaushal, Nadia Sofía Macias Vera, Robin Bollache, Clément Honorez, Alain Cagna, and Wiebke Drenckhan, "Microfluidic thin film pressure balance for the study of complex thin films," Lab Chip 21, 412-420 (2021).

[16] R. Bruinsma, "Theory of hydrodynamic convection in soap films," Physica A 216, 59 - 76 (1995).

[17] Alice Gros, Adrien Bussonnière, Sanjiban Nath, and Isabelle Cantat, "Marginal regeneration in a horizontal film: Instability growth law in the nonlinear regime," Phys. Rev. F 6, 024004 (2021).

[18] H. N. Stein, "On marginal regeneration," Adv. Colloid Interface Sci. 34, 175 - 190 (1991).

[19] Vincent Adriaan Nierstrasz and Gert Frens, "Marginal regeneration in thin vertical liquid films," Journal of Colloid and Interface Science 207, 209 - 217 (1998).

[20] Jonas Miguet, Amincissement et stabilité de bulles de surface, Ph.D. thesis, Université Paris-Saclay (2019).

[21] Yiran Zhang and Vivek Sharma, "Thickness-dependent phase transition drives nanoridge-to-mesa instability in micellar freestanding films," Langmuir 34, 7922-7931 (2018).

[22] V Chandran Suja, A Hadidi, A Kannan, and GG Fuller, "Axisymmetry breaking, chaos, and symmetry recovery in bubble film thickness profiles due to evaporation-induced marangoni flows," Phys. Fluids 33, 012112 (2021).

[23] Paul Stevenson, "Remarks on the shear viscosity of surfaces stabilised with soluble surfactants," J. Colloid Interface Sci. 290, $603-606$ (2005).

[24] W. Drenckhan, H. Ritacco, A. Saint-Jalmes, A. Saugey, P. McGuinness, A. Van der Net, D. Langevin, and D. Weaire, "Fluid dynamics of rivulet flow between plates," Phys. Fluids 19, 102101 (2007).

[25] L. Champougny, J. Miguet, R. Henaff, F. Restagno, F. Boulogne, and E. Rio, "Influence of evaporation on soap film rupture," Langmuir 34, 3221-3227 (2018).

[26] J. Seiwert, B. Dollet, and I. Cantat, "Theoretical study of the generation of soap films: role of interfacial visco-elasticity," J. Fluid Mech. 739, 124-142 (2014).

[27] Alexander Oron, Stephen H Davis, and S George Bankoff, "Long-scale evolution of thin liquid films," Rev. Mod. Phys 69, 931 (1997).

[28] Tomas Bohr and Bernhard Scheichl, "Surface tension and energy conservation in a moving fluid," Phys. Rev. F 6, L052001 (2021), 2011.11403.

[29] George Pólya and Gábor Szegö, Isoperimetric Inequalities in Mathematical Physics.(AM-27), Volume 27 (Princeton University Press, 1951). 